\title{
ON SINC QUADRATURE APPROXIMATIONS OF FRACTIONAL POWERS OF REGULARLY ACCRETIVE OPERATORS
}

\author{
ANDREA BONITO, WENYU LEI, AND JOSEPH E. PASCIAK
}

\begin{abstract}
We consider the finite element approximation of fractional powers of regularly accretive operators via the Dunford-Taylor integral approach. We use a sinc quadrature scheme to approximate the Balakrishnan representation of the negative powers of the operator as well as its finite element approximation. We improve the exponentially convergent error estimates from [A. Bonito, J. E. Pasciak, IMA J. Numer. Anal. (2016) 00, 1-29] by reducing the regularity required on the data. Numerical experiments illustrating the new theory are provided.
\end{abstract}

\section{Introduction.}

Let $Y \subset X$ be complex valued Hilbert spaces with $Y$ dense in $X$. Let $A(\cdot, \cdot)$ be a bounded and coercive sesquilinear form on $Y$. Following [17] (see, Section 2) there is a uniquely defined unbounded operator on $X$ denoted by $A$ with domain $D(A) \subset Y$. The fractional powers of $A$ are given by the Balakrishnan integral [4, 17. defined for $\beta \in(0,1)$ and $f \in X$ :

$$
u=A^{-\beta} f=\frac{\sin (\pi \beta)}{\pi} \int_{0}^{\infty} \mu^{-\beta}(\mu I+A)^{-1} f d \mu .
$$

After the change of variable $\mu=e^{y}$, we approximate the resulting integral using a truncated equally spaced quadrature (sinc quadrature [19]) given by

$$
u_{k}:=\mathcal{Q}_{k}^{-\beta}(A) f:=\frac{k \sin (\pi \beta)}{\pi} \sum_{\ell=-M}^{N} e^{(1-\beta) y_{\ell}}\left(e^{y_{\ell}} I+A\right)^{-1} f,
$$

where $k>0$ is a real number and $N$ and $M$ are positive integers chosen to be on the order of $1 / k^{2}$ (see Remark 3.1).

The fractional powers $A^{s}$ (with domain $D\left(A^{s}\right)$ ) are well defined for $s \geq 0$, see [18. It is a consequence of the coercivity of $A(\cdot, \cdot)$ that the natural norm on $D\left(A^{s}\right)$, namely $\left(\|v\|_{X}^{2}+\left\|A^{s} v\right\|_{X}\right)^{1 / 2}$ is equivalent to the norm

$$
\|v\|_{D\left(A^{s}\right)}:=\left\|A^{s} v\right\|_{X} .
$$

The first result of this note, Theorem 3.2 shows that the quadrature error goes exponentially to zero when measured in a scale of the above norms. Specifically, for any $s \in[0, \beta)$ and $t \geq 0$,

$$
\left\|u-\mathcal{Q}_{k}^{\beta}(A) f\right\|_{D\left(A^{s+t}\right)} \leq C e^{-c / k}\|f\|_{D\left(A^{t}\right)}
$$

Date: February 5, 2018.

1991 Mathematics Subject Classification. 65N30, 35S15, 65N15, 65R20, 65N12.

AB is partially supported by NSF grant DMS-1254618.

WL is partially supported by NSF grant DMS- 1254618 . 
with $C$ and $c$ not depending on $k$. The result of this paper requires less regularity on $f$ than that of [10] which is essentially of the form (for $s \geq 0$ )

$$
\left\|\left(u-\mathcal{Q}_{k}^{\beta}(A) f\right)\right\|_{D\left(A^{s}\right)} \leq C e^{-c / k}\|f\|_{D\left(A^{s}\right)} .
$$

In the remainder of the paper, we focus on fractional problems with homogeneous Dirichlet boundary conditions. We avoid the non-homogeneous case (see [3] for a discussion of one possible definition for fractional problems with non-homogeneous Dirichlet and Neumann boundary conditions). Let $\Omega \subset \mathbb{R}^{d}$ be a bounded domain with Lipschitz boundary $\Gamma:=\partial \Omega$. We assume that there are two open sets (with respect to Lebesgue measure in $\left.\mathbb{R}^{d-1}\right) \Gamma_{D}$ (Dirichlet) and $\Gamma_{N}$ (Neumann) such that $\Gamma_{D} \cap \Gamma_{N}=\emptyset$ and $\overline{\Gamma_{D} \cup \Gamma_{N}}=\Gamma$. We additionally assume that $\Gamma_{D} \neq \emptyset$. We define the complexed valued functional space

$$
\mathbb{V}:=\left\{v \in H^{1}(\Omega): v=0 \text { on } \Gamma_{D}\right\} \subset H^{1}(\Omega)
$$

and set

$$
X:=L^{2}(\Omega), \quad \text { and } \quad Y:=\mathbb{V} .
$$

Also for all $u, v \in \mathbb{V}$ we consider

$$
A(u, v):=\int_{\Omega} \mathbf{A} \nabla u \cdot \nabla \bar{v}+\mathbf{b}_{1} \cdot \nabla u \bar{v}+u \mathbf{b}_{2} \cdot \nabla \bar{v}+c u \bar{v} d x
$$

where $\bar{v}$ denotes the complex conjugate of $v$. The coefficients $\mathbf{A} \in L^{\infty}\left(\Omega, \mathrm{GL}\left(\mathbb{R}^{d}\right)\right)$, $\mathbf{b}_{1}, \mathbf{b}_{2} \in L^{\infty}\left(\Omega, \mathbb{R}^{d}\right), c \in L^{\infty}(\Omega)$ are assumed to be such that the form $A(\cdot, \cdot)$ is coercive and bounded on $\mathbb{V}$.

The numerical approximation of (1) with $X, Y, A(\cdot, \cdot)$ and $\mathbb{V}$ as in (5)-(6). is defined as follows. One starts by introducing a finite element space $\mathbb{V}_{h}$. The general framework of Section 2 is used to define the discrete fractional power $A_{h}^{\beta}$ for $X=$ $Y=\mathbb{V}_{h}$ and $A(\cdot, \cdot)$ as in (6). The (semi-discrete) finite element approximation of $u=A^{-\beta} f$ is then defined by $u_{h}=A_{h}^{-\beta} \pi_{h} f$ where $\pi_{h}$ denotes the $L^{2}(\Omega)$ projection onto $\mathbb{V}_{h}$. Of course,

$$
u_{h}=A_{h}^{-\beta} \pi_{h} f=\frac{\sin (\pi \beta)}{\pi} \int_{0}^{\infty} \mu^{-s}\left(\mu I+A_{h}\right)^{-1} \pi_{h} f d \mu .
$$

The fully discrete approximation is then defined by $u_{h, k}=\mathcal{Q}_{k}^{-\beta}\left(A_{h}\right) \pi_{h} f$ and our goal is to estimate the error $u-u_{h, k}$.

We shall provide error estimates in Sobolev norms. To this end, we define

$$
\mathbb{H}^{r}(\Omega):= \begin{cases}\left(L^{2}(\Omega), H_{0}^{1}(\Omega)\right)_{2, r}, & \text { for } r \in[0,1], \\ H^{r}(\Omega) \cap H_{0}^{1}(\Omega), & \text { for } r \in[1,2],\end{cases}
$$

with $(\cdot, \cdot)_{2, r}$ denoting interpolation using the real method. We assume elliptic regularity with index $\alpha \in(0,1]$ (see, Assumption 4.1). When this condition holds,

$$
D\left(A^{s / 2}\right)=\mathbb{H}^{s}(\Omega), \quad \text { for } s \in[0,1+\alpha]
$$

and their norms are equivalent. Indeed, for $s \in[0,1)$, this follows from Theorem 3.1 of [17, $s=1$ is proved in [2] and $s \in(1,1+\alpha]$ is given by Theorem 6.4 of [10].

We shall not assume artificial smoothness of the solution $u=A^{-\beta} f$. Instead, we assume smoothness on $f$ and use the smoothing properties of the operator $A^{-\beta}$ and (9) to conclude regularity for $u$. Note that for $t \geq 0, u$ is in $D\left(A^{t+\beta}\right)$ if and only if $f$ is in $D\left(A^{t}\right)$ with equal norms. In all cases, we assume that $f$ is in $X:=L^{2}(\Omega)$. 
Estimates for the error between $u$ and $u_{h}$ in the $\mathbb{H}^{r}(\Omega)$ norms for $r \in[0,1]$ were provided in [10] and are also discussed in Subsection 4.3. We shall apply Theorem 3.2 to the derive bounds for the error $\left\|u_{h}-u_{h, k}\right\|_{\mathbb{H}^{r}(\Omega)}$ and obtain (Theorem 4.2

$$
\left\|u_{h}-u_{h, k}\right\|_{\mathbb{H}^{r}(\Omega)} \preceq e^{-\pi^{2} /(2 k)}\|f\|_{\mathbb{H}^{\max \left(r+2 \alpha^{*}-2 \beta, 0\right)}(\Omega)}
$$

with $2 \alpha^{*}=\alpha+\min (\alpha, 1-r)$. Note that the norm on $f$ appearing above is always controlled by the norm on $f$ needed in [10] to obtain an $h^{\epsilon}(0<\epsilon \ll 1)$ convergence bound for $\left\|u-u_{h}\right\|_{\mathbb{H}^{r}(\Omega)}$ (see Subsection 4.3) so the exponential convergence of the sinc approximation is achieved without additional assumptions on $f$.

This improves the estimates obtained for the sinc approximation in 10. For example, if $\Omega$ is convex and the coefficients of $A(\cdot, \cdot)$ are smooth, $D\left(A^{s}\right)=\mathbb{H}^{2 s}(\Omega)$ and the norms $\left\|A^{s} \cdot\right\|$ and $\|\cdot\|_{\mathbb{H}^{2 s}(\Omega)}$ are equivalent for $s \in[0,1]$. As shown in [10, the energy norm error, $\left\|u-u_{h}\right\|_{H^{\beta}(\Omega)}$ is $O\left(h^{2-\beta}\right)$ up to a logarithm of $h^{-1}$ for $f \in \mathbb{H}^{2-2 \beta}(\Omega)$. In this case, 10 implies exponential convergence of the sinc approximation in the energy norm while (4) requires $f \in \mathbb{H}^{\beta}(\Omega)$ which corresponds to more regularity when $\beta>2 / 3$.

We refer to [7] for a review of different numerical methods tailored to fractional diffusion. To the best of our knowledge, besides the above mentioned works, there are no alternative numerical method for the approximation of fractional powers of general regularly accretive operators. However, several methods are available when the operator $A$ is real symmetric. We refer to [16, 15, 22] for methods based on expansions using the eigenpairs of the discretized operator and to [21] as well as to [20, 5] where approximations of the "Neumann to Dirichlet" map of an extended problem is advocated. In [1, 13, numerical approximation of the integral definition of the fractional laplacian is considered. It is worth mentioning that in this context, the recent work [8] is also based on sinc quadrature approximations of a DunfordTaylor representation.

The outline of this note is as follows. In Section 2, we review the definition and properties of fractional powers of the regularly accretive operator $A$. In Section 3 . we prove abstract estimates for the error in the sinc quadrature error $u_{h}-u_{h, k}$ showing exponential convergence in the norms of $D\left(A^{s}\right)$, for $s \geq 0$ under appropriate regularity conditions on $f$. We consider the setting described by (5)-(6) in Section 4 and provide error estimates for $u-u_{h, k}$ by combining the estimates for the error $u-u_{h}$ given by [10] and the results of Section 3 . We provide numerical illustrations of the improved theory in Section 5 .

We write $a \preceq b$ to mean $a \leq C b$, with a constant $C$ that does not depend on $a$, $b$, or the discretization parameters. Finally, $a \approx b$ indicates $a \preceq b$ and $b \preceq a$.

ACKnowledgment. The authors would like to thank R.H. Nochetto for pointing out the possible sub-optimality in [10, thereby prompting the current analysis.

\section{Fractional Powers of Regularly Accretive Operators.}

As in the introduction, we consider complex valued Hilbert spaces with $Y$ continuously and densely imbedded in $X$ and a bounded and coercive sesquilinear form $A(\cdot, \cdot)$. This means that there are constants $c_{0}$ and $C_{0}$ satisfying

$$
\mathfrak{R e}(A(v, v)) \geq c_{0}\|v\|_{Y}^{2}, \quad \text { for all } v \in Y,
$$


and

$$
|A(u, v)| \leq C_{0}\|u\|_{Y}\|v\|_{Y}, \quad \text { for all } u, v \in Y .
$$

Such a sesquilinear form $A(\cdot, \cdot)$ is called regular (cf. [17]). By possibly rescaling the norm in $X$, we may assume that

$$
\|y\|_{X} \leq\|y\|_{Y}, \quad \text { for all } y \in Y .
$$

To simplify the notation, we denote $\|v\|:=\|v\|_{X}$ for $v \in X$ and $\|\mathcal{A}\|$ to be the operator norm of $\mathcal{A}$ when $\mathcal{A}$ is a bounded operator from $X$ into $X$.

Following [17, there is a uniquely defined unbounded operator on $X$ denoted by $A$ with domain $D(A) \subset Y$ defined as follows. Invoking the Lax-Milgram Theorem, we define the one-to-one solution operator $T: X \rightarrow Y$ satisfying

$$
A(T f, \phi)=(f, \phi), \quad \text { for all } \phi \in Y .
$$

The unbounded operator $A$ is defined by $A w:=T^{-1} w$ for $w$ in $D(A):=\operatorname{Range}(T)$. The operator $A$ is a closed densely defined operator on $X$ with domain $D(A)$. Such an operator is said to be regularly accretive (cf. [17]).

For regularly accretive operators, there exists $\omega \in[0, \pi / 2)$ such that the spectrum of $A$ is contained in the sector $S_{\omega}:=\{z \in \mathbb{C}:|\arg z| \leq \omega\}$. We also have the following estimate for the resolvent $R_{z}(A):=(A-z I)^{-1}$

$$
\left\|R_{z}(A) f\right\| \leq(\sin (\pi / 2-\omega))^{-1}|z|^{-1}\|f\|, \quad \text { for } \mathfrak{R e}(z)<0 .
$$

Also, if $z$ is negative, $\left\|R_{z}(A) f\right\| \leq|z|^{-1}\|f\|$, i.e., $A$ is M-accretive. The above bounds are a consequence of Theorem 2.2 of [17. For later use, we also note that the coercivity assumption (11) and (13) implies that for $\mathfrak{R e}(z) \leq c_{0} / 2$,

$$
\left\|R_{z}(A) f\right\| \leq\left\|R_{z}(A) f\right\|_{Y} \leq \frac{2}{c_{0}}\|f\| .
$$

2.1. Fractional Powers. For $\beta \in(0,1)$, the negative fractional powers $A^{-\beta}$ of a regularly accretive operator $A$ are defined by (1). It follows from (15) and (16) that the integral (1) is Bochner integrable in $X$ and so $A^{-r}$ is a bounded operator on $X$. When $r$ is non-negative and not an integer with $n-1<r<n$, then

$$
D\left(A^{r}\right):=\left\{x \in X: A^{r-n} x \in D\left(A^{n}\right)\right\}, \quad A^{r} x=A^{n} A^{r-n} x .
$$

We shall make use of the following commutivity proproperties involving fractional powers and the resolvent, see e.g. [18.

(a) For $r \geq 0$ and $\beta \in(0,1)$,

$$
A^{-\beta} A^{r} x=A^{r-\beta} x=A^{r} A^{-\beta} x, \quad \text { for all } x \in D\left(A^{r}\right) .
$$

(b) For $z \in \rho(A)$ and $r \geq 0, R_{z}(A): D\left(A^{r}\right) \rightarrow D\left(A^{r+1}\right)$ and

$$
A^{r} R_{z}(A) x=R_{z}(A) A^{r} x, \quad \text { for all } x \in D\left(A^{r}\right) .
$$

2.2. Interpolation Scales. Since a regularly accretive operator is M-accretive, Corollary 4.3.6 of [18] shows that for $s \in(0,1)$,

$$
[X, D(A)]_{s}=D\left(A^{s}\right)
$$

with $[\cdot, \cdot]_{s}$ denoting the interpolation scale using the complex method. Corollary 2.1.8 of [18] then implies that

$$
\left\|A^{s} v\right\| \preceq\|v\|_{\left[L^{2}(\Omega), D(A)\right]_{s}} \preceq\|A v\|^{s}\|v\|^{1-s}, \quad \text { for all } v \in D(A) .
$$




\section{Sinc Approximations to $A^{-\beta}$.}

In this section, we revisit the sinc approximation technique developed in 10 (see, (2) ) and provide an abstract theorem which weakens the regularity required on $f$ to achieve an exponential rate of convergence.

As anticipated in the introduction, we use the change of variable $\mu:=e^{y}$ in (1), i.e.,

$$
A^{-\beta}=\frac{\sin (\pi \beta)}{\pi} \int_{-\infty}^{\infty} e^{(1-\beta) y}\left(e^{y} I+A\right)^{-1} d y .
$$

For any positive integers $N$ and $M$ and a positive quadrature step $k$, we define the sinc approximation of $A^{-\beta}$ by 2 which corresponds to a truncated equally spaced quadrature approximation to 20). Notice that to simplify the notation, we do not specify the dependency on $M$ and $N$. In any event, in practice both $M$ and $N$ are functions of $k$ (see Remark 3.1).

3.1. Error Analysis. The analysis for the sinc approximation error involves the analyticity and decay properties of the function

$$
F(z ; \theta, \eta):=e^{(1-\beta) z}\left(A^{s}\left(e^{z} I+A\right)^{-1} \theta, \eta\right) .
$$

on the band

$$
z \in \mathcal{S}_{\pi / 2}:=\{z \in \mathbb{C}:|\mathfrak{I m}(z)| \leq \pi / 2\} .
$$

Here $(\cdot, \cdot)$ denotes the inner product on $X$ and $\theta, \eta$ are fixed in $X$. The decay properties are addressed in the following lemma.

Lemma 3.1 (Integrand Estimate). Let $s$ be in $[0, \beta)$ and $\delta$ be non-negative. For $z \in \mathcal{S}_{\pi / 2}$

$$
\left\|e^{(1-\beta) z} A^{s}\left(e^{z} I+A\right)^{-1}\right\| \preceq \begin{cases}e^{(s-\beta) \Re \mathfrak{R}(z)} & : \quad \text { when } \mathfrak{R e}(z)>0 . \\ e^{(1-\beta) \mathfrak{R e}(z)} & : \quad \text { when } \mathfrak{R e}(z) \leq 0 .\end{cases}
$$

The hidden constant depends only on $\beta, s, c_{0}$ and $C_{0}$.

Proof. We fix $z \in \mathcal{S}_{\pi / 2}$ and $\theta \in X$. As $-e^{z}$ is in $\rho(A)$ (in fact, $\mathfrak{R e}\left(-e^{z}\right)<0$ ), $\left(e^{z} I+A\right)^{-1} \theta$ is in $D(A)$. Applying 19 gives

$$
\left\|A^{s}\left(e^{z} I+A\right)^{-1} \theta\right\| \preceq\left\|\left(e^{z} I+A\right)^{-1} \theta\right\|^{1-s}\left\|A\left(e^{z} I+A\right)^{-1} \theta\right\|^{s} .
$$

We apply (15) and (16) to obtain

$$
\left\|\left(e^{z} I+A\right)^{-1} \theta\right\| \leq\|\theta\| \begin{cases}(\sin (\pi / 2-\omega))^{-1} e^{-\mathfrak{R e}(z),} & \text { when } \mathfrak{R e}(z)>0, \\ c_{0} / 2, & \text { when } \mathfrak{R e}(z) \leq 0 .\end{cases}
$$

Also 150 implies that

$$
\left\|e^{z}\left(e^{z} I+A\right)^{-1}\right\| \leq(\sin (\pi / 2-\omega))^{-1}, \quad \text { for all } z \in \mathcal{S}_{\pi / 2} .
$$

and hence

$$
\left\|A\left(e^{z} I+A\right)^{-1}\right\|=\left\|I-e^{z}\left(e^{z} I+A\right)^{-1}\right\| \leq 1+(\sin (\pi / 2-\omega))^{-1} .
$$

Combining the above estimates gives

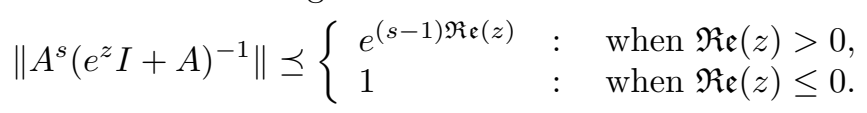

The lemma follows from the above estimates and the trivial estimate,

$$
\left|e^{(1-\beta) z}\right|=e^{(1-\beta) \Re \mathfrak{R}(z)} .
$$


Now by 222 and 23], for $z \in \mathcal{S}_{\pi / 2}$,

$$
\begin{aligned}
\left|\frac{d}{d z} F(z ; \theta, \eta)\right| \leq & \left|e^{(2-\beta) z}\left(A^{s}\left(e^{z} I+A\right)^{-2} \theta, \eta\right)\right|+(1-\beta)|F(z ; \theta, \eta)| \\
& \preceq\|\theta\|\|\eta\| \begin{cases}e^{(1+s-\beta) \mathfrak{R e}(z)} & : \quad \text { when } \mathfrak{R e}(z)>0, \\
e^{(1-\beta) \mathfrak{R e}(z)} & : \quad \text { when } \mathfrak{R e}(z) \leq 0,\end{cases}
\end{aligned}
$$

i.e., $F(. ; \theta, \eta)$ is analytic on $\mathcal{S}_{\pi / 2}$ for each $\theta$ and $\eta$ in $X$.

We are now in position to prove our result which bounds the quadrature error.

Theorem 3.2 (Sinc Quadrature Error). For $M, N>0$ integers, and $k>0$, let $\mathcal{Q}_{k}^{-\beta}(A)$ be defined by (2). Given $f \in D\left(A^{t}\right)$ with $t \geq 0$ and $-t \leq s<\beta$, then

$$
\begin{gathered}
\left\|\left(A^{-\beta}-\mathcal{Q}_{k}^{-\beta}(A)\right) f\right\|_{D\left(A^{s+t}\right)} \preceq\left[\left(\sinh \left(\pi^{2} /(2 k)\right)\right)^{-1} e^{-\pi^{2} /(2 k)}\right. \\
\left.+e^{-\left(\beta-s^{+}\right) N k}+e^{-(1-\beta) M k}\right]\|f\|_{D\left(A^{t}\right)},
\end{gathered}
$$

where $s^{+}=\max (0, s)$ and the hidden constant depends only on $\beta, s, c_{0}$ and $C_{0}$.

Proof. Using (18), 17) and the definition of the norm in 24, 24) can be rewritten

$$
\left\|\left(A^{-\beta}-\mathcal{Q}_{k}^{-\beta}(A)\right) A^{s+t} f\right\| \preceq C(k)\left\|A^{t} f\right\|, \quad \text { for all } f \in D\left(A^{t}\right)
$$

with $C(k)$ denoting the expression in brackets on the right hand side of (24). Since $A^{t}$ is a one to one map of $D\left(A^{t}\right)$ onto $X$ and $A^{s}$ is a bounded operator when $s<0$, it is suffices to show that

$$
\left|\left(\left(A^{-\beta}-\mathcal{Q}_{k}^{-\beta}(A)\right) A^{s^{+}} \theta, \eta\right)\right| \preceq C(k), \quad \text { for all } \theta, \eta \in X
$$

with $\|\theta\|=\|\eta\|=1$.

Extending the sum in 25 to a sum over all integers and applying the triangle inequality gives

$$
\begin{aligned}
\left|\left(\left(A^{-\beta}-\mathcal{Q}_{k}^{-\beta}(A)\right) A^{s^{+}} \theta, \eta\right)\right| \leq \mid & \int_{-\infty}^{\infty} F(y ; \theta, \eta) d y-k \sum_{\ell=-M}^{N} F(\ell k ; \theta, \eta) \mid \\
& +\left|k \sum_{\ell>N} F(\ell k ; \theta, \eta)\right|+\left|k \sum_{\ell<-M} F(\ell k ; \theta, \eta)\right| .
\end{aligned}
$$

1 For the second sum on the right hand side of 26, applying Lemma 3.1 gives

$$
k \sum_{\ell>N}|F(\ell k ; \theta, \eta)| \preceq \int_{N k}^{\infty} e^{\left(s^{+}-\beta\right) y} d y=e^{\left(s^{+}-\beta\right) N k} /\left(\beta-s^{+}\right) .
$$

Similarly,

$$
k \sum_{\ell<-M}|F(\ell k ; \theta, \eta)| \preceq \int_{-\infty}^{-M k} e^{(1-\beta) y} d y=e^{(\beta-1) M k} /(1-\beta) .
$$

2 For first sum on the right hand side of (26), we invoke Theorem 2.20 in [19], which states that

$$
\left|\int_{-\infty}^{\infty} F(y ; \theta, \eta)-k \sum_{\ell=-\infty}^{\infty} F(\ell k ; \theta, \eta)\right| \leq \frac{R}{2 \sinh \left(\pi^{2} /(2 k)\right)} e^{-\pi^{2} /(2 k)}
$$


provided that

$$
\int_{-\pi / 2}^{\pi / 2}|F(t+i y ; \theta, \eta)| d y \preceq 1, \quad \text { for all } t \in \mathbb{R}
$$

and $R$ is a constant such that

$$
\int_{-\infty}^{\infty}(|F(y-i \pi / 2 ; \theta, \eta)|+|F(y+i \pi / 2 ; \theta, \eta)|) d y \leq R
$$

Both conditions follows from Lemma 3.1. In particular,

$$
R=2 C\left(\left(\beta-s^{+}\right)^{-1}+(1-\beta)^{-1}\right),
$$

where $C$ is the constant hidden in estimate (21).

3 The desired estimate (24) for $A$ is obtained upon gathering the estimates derived in steps 1 and 2 .

Remark 3.1 (Exponential Decay). In practice, we advocate to balance the three exponentials on the right hand side of (24), thereby imposing

$$
\pi^{2} /(2 k) \approx\left(\beta-s^{+}\right) k N \approx(1-\beta) k M .
$$

Thus, given $k>0$, we set

$$
N=\left\lceil\frac{\pi^{2}}{2\left(\beta-s^{+}\right) k^{2}}\right\rceil \quad \text { and } \quad M=\left\lceil\frac{\pi^{2}}{2(1-\beta) k^{2}}\right\rceil,
$$

which leads to

$$
\begin{aligned}
& \left\|\left(A^{-\beta}-\mathcal{Q}_{k}^{-\beta}(A)\right) \pi f\right\|_{D\left(A^{s+t}\right)} \\
& \quad \preceq\left[\frac{1}{\beta-s^{+}}+\frac{1}{1-\beta}\right]\left[\frac{e^{-\pi^{2} /(2 k)}}{\sinh \left(\pi^{2} /(2 k)\right)}+e^{-\pi^{2} /(2 k)}\right]\|f\|_{D\left(A^{t}\right)} .
\end{aligned}
$$

Note that the coefficient on the right hand side above asymptotically behaves like

$$
\left[\frac{1}{\beta-s^{+}}+\frac{1}{1-\beta}\right] e^{-\pi^{2} /(2 k)} \quad \text { as } \quad k \rightarrow 0 .
$$

This choice will be refer to as the "balanced" scheme. Another possibility is to simply take $k^{-2} \sim N=M$, which also lead to exponential decay but, as we shall see in Section 5 , is less efficient.

\section{The Numerical Approximation of (5)- $(6)$.}

For the remainder of this paper, we focus on the example described by (5)-(6). The coercivity and boundedness assumptions made in the introduction guarantee that (11) and (12) hold with $Y=\mathbb{V}$ so that the operator $A$ associated with (6) and its fractional powers are defined in Section 2.1.

4.1. The Finite Element Approximation. We now assume that $\Omega$ is a polyhedon. Let $\left\{\mathcal{T}_{h}\right\}_{h>0}$ be a sequence of conforming subdivisions made of simplices with maximal mesh size $h<1$. We further assume that $\left\{\mathcal{T}_{h}\right\}$ are shape-regular and quasi-uniform (cf. [14]) with constants independent of $h$ and the triangulation matches the partitioning $\Gamma=\overline{\Gamma_{D} \cup \Gamma_{N}}$. Let $\mathbb{V}_{h}$ be the space of continuous piecewise linear finite element functions subordinate to $\mathcal{T}_{h}$ and let $\pi_{h}$ be the $L^{2}$ orthogonal projector onto $\mathbb{V}_{h}$. The semi-discrete approximation $u_{h}$ and the fully discrete approximation $u_{h, k}$ are defined as in the introduction. 
Before going further, we note that the quasi-uniformity assumption is required to guarantee the $H^{1}(\Omega)$ stability of $\pi_{h}$, namely

$$
\left\|\pi_{h} v\right\|_{H^{1}(\Omega)} \preceq\|v\|_{H^{1}(\Omega)} .
$$

The quasi-uniformity assumption can be relaxed, for instance to allow certain grading condition on $\mathcal{T}_{h}$; see [12, 11, 6]. The $H^{1}$ stability estimate 30 implies, by interpolation, that for $r \in[0,1]$

$$
\left\|\pi_{h} v\right\|_{\mathbb{H}^{r}(\Omega)} \preceq\|v\|_{\mathbb{H}^{r}(\Omega)} .
$$

4.2. Elliptic regularity and finite element error estimates. We define $\mathbb{H}_{a}^{-1}$ to be the set of bounded anti-linear functionals on $\mathbb{V}$. As usual, $L^{2}(\Omega)$ can be imbedded in $\mathbb{H}_{a}^{-1}$ by identifying $f \in L^{2}(\Omega)$ with the functional

$$
\langle F, \phi\rangle=(f, \phi), \quad \text { for all } \phi \in \mathbb{V}
$$

with $\langle\cdot, \cdot\rangle$ denoting the anti-linear functional/function pairing. We define the spaces $\mathbb{H}_{a}^{r}$ to $r \in[-1,0)$ by setting

$$
\mathbb{H}_{a}^{r}=\left(\mathbb{H}_{a}^{-1}, L^{2}(\Omega)\right)_{r+1,2} .
$$

It follows that $T: L^{2}(\Omega) \rightarrow D(A)$ extends to a bounded antilinear map of $\mathbb{H}_{a}^{-1} \rightarrow \mathbb{V}$ defined by replacing $(f, \phi)$ with $\langle F, \phi\rangle$.

The adjoint operator $T^{*}: L^{2}(\Omega) \rightarrow \mathbb{V}$ is defined analogously to $T$, i.e.,

$$
A\left(\phi, T^{*} g\right)=(\phi, g), \quad \text { for all } \phi \in \mathbb{V} \text {. }
$$

In this case, $T^{*} g$ is a linear functional and extends to $\mathbb{H}_{l}^{-1}$, the set of bounded linear functionals on $\mathbb{V}$. As above, we define

$$
\mathbb{H}_{l}^{r}=\left(\mathbb{H}_{l}^{-1}, L^{2}(\Omega)\right)_{r+1,2} .
$$

The analysis of the error between $u$ and $u_{h}$ relies on the regularity of $T$ and $T^{*}$ described in the following assumption.

Assumption 4.1. There exists $\alpha \in(0,1]$ such that:

(a) $T$ is an isomorphism from $\mathbb{H}_{a}^{-1+r}(\Omega)$ to $\mathbb{H}^{1+r}(\Omega)$ for any $r \in(0, \alpha]$.

(b) $T^{*}$ is an isomorphism from $\mathbb{H}_{l}^{-1+r}(\Omega)$ to $\mathbb{H}^{1+r}(\Omega)$ for any $r \in(0, \alpha]$.

Let $P_{h}: \mathbb{V} \rightarrow \mathbb{V}_{h}$ denote the elliptic projection defined by

$$
A\left(P_{h} u, v\right)=A(u, v), \quad \text { for all } v \in \mathbb{V}_{h} .
$$

We note that it is a consequence of Assumption 4.1 and standard finite element error analysis arguments that for $r \in(0, \alpha)$

$$
\left\|\left(I-P_{h}\right) u\right\|_{H^{1}(\Omega)} \preceq h^{r_{1}}\|u\|_{H^{1+r_{1}(\Omega)}}, \quad \text { for all } u \in H^{1+r_{1}}(\Omega) \cap \mathbb{V}
$$

and

$$
\left\|\left(I-P_{h}\right) u\right\|_{\mathbb{H}^{1-r_{2}(\Omega)}} \preceq h^{r_{2}}\|u\|_{H^{1}(\Omega)}, \quad \text { for all } u \in \mathbb{V}
$$

From the equivalence result in [17] and (31), we deduce that the discrete norms

$$
\left\|v_{h}\right\|_{\mathbb{H}^{r}(\Omega)} \approx\left\|A_{h}^{r / 2} v_{h}\right\|, \quad \text { for all } v_{h} \in \mathbb{V}_{h},
$$

are equivalent for $r \in[0,1)$. This equivalence holds also for the adjoint operator $A_{h}^{*}$ and the case $r=1$ follows from Assumption 4.1 (see, [10, Theorem 6.5]).

When $r>1$, we have the following lemma. 
Lemma 4.1. Let $r \in(1,1+\alpha]$ where is $\alpha$ the regularity index in Assumption 4.1. For $v \in \mathbb{H}^{r}(\Omega)$, there holds

$$
\left\|A_{h}^{r / 2} \pi_{h} v\right\| \preceq\|v\|_{\mathbb{H}^{r}(\Omega)} .
$$

Proof. We first show that

$$
\left\|A_{h}^{r / 2} P_{h} v\right\| \preceq\|v\|_{\mathbb{H}^{r}(\Omega)} .
$$

In fact,

$$
\begin{aligned}
\left\|A_{h}^{r / 2} P_{h} v\right\| & =\sup _{\theta_{h} \in \mathbb{V}_{h}} \frac{\left(A_{h}^{r / 2} P_{h} v, \theta_{h}\right)}{\left\|\theta_{h}\right\|} \\
& =\sup _{\theta_{h} \in \mathbb{V}_{h}} \frac{A\left(P_{h} v,\left(A_{h}^{*}\right)^{r / 2-1} \theta_{h}\right)}{\left\|\theta_{h}\right\|}=\sup _{\theta_{h} \in \mathbb{V}_{h}} \frac{A\left(v,\left(A_{h}^{*}\right)^{r / 2-1} \theta_{h}\right)}{\left\|\theta_{h}\right\|} .
\end{aligned}
$$

Then we let $\phi_{h}=\left(A_{h}^{*}\right)^{r / 2-1} \theta_{h}$ and apply the discrete norm equivalence 34 for $A_{h}^{*}$ to yield

$$
\begin{aligned}
\left\|A_{h}^{r / 2} P_{h} v\right\| & =\sup _{\phi_{h} \in \mathbb{V}_{h}} \frac{A\left(v, \phi_{h}\right)}{\left\|\left(A_{h}^{*}\right)^{1-r / 2} \phi_{h}\right\|} \preceq \sup _{\phi_{h} \in \mathbb{V}_{h}} \frac{A\left(v, \phi_{h}\right)}{\left\|\phi_{h}\right\|_{\mathbb{H}^{1-r / 2}(\Omega)}} \\
& =\sup _{\phi_{h} \in \mathbb{V}_{h}} \frac{\left(A^{r / 2} v,\left(A^{*}\right)^{1-r / 2} \phi_{h}\right)}{\left\|\phi_{h}\right\|_{\mathbb{H}^{1-r / 2}(\Omega)}} \leq \sup _{\phi \in \mathbb{H}^{1-r / 2}(\Omega)} \frac{\left(A^{r / 2} v,\left(A^{*}\right)^{1-r / 2} \phi\right)}{\|\phi\|_{\mathbb{H}^{1-r / 2}(\Omega)}} \\
& \preceq\|v\|_{\mathbb{H}^{r}(\Omega)} .
\end{aligned}
$$

We also need the inverse estimate. Note that for $v_{h} \in \mathbb{V}_{h}$,

$$
\left\|A_{h}^{1 / 2} v_{h}\right\| \approx\left\|v_{h}\right\|_{H^{1}(\Omega)} \preceq h^{-1}\left\|v_{h}\right\| .
$$

This implies that for $s \in[0,1]$,

$$
\left\|A_{h}^{s / 2} v_{h}\right\| \preceq h^{-s}\left\|v_{h}\right\| .
$$

Now, we invoke (35) together with (30), 32) and the inverse inequality (36) to conclude that

$$
\begin{aligned}
\left\|A_{h}^{r / 2} \pi_{h} v\right\| & \leq\left\|A_{h}^{r / 2} P_{h} v\right\|+\left\|A_{h}^{r / 2}\left(\pi_{h}-P_{h}\right) v\right\| \\
& \preceq\|v\|_{\mathbb{H}^{r}(\Omega)}+h^{1-r}\left\|\left(\pi_{h}-P_{h}\right) v\right\|_{H^{1}(\Omega)} \\
& \preceq\|v\|_{\mathbb{H}^{r}(\Omega)}+h^{1-r}\left\|\left(I-P_{h}\right) v\right\|_{H^{1}(\Omega)} \preceq\|v\|_{\mathbb{H}^{r}(\Omega)} .
\end{aligned}
$$

4.3. The error between $u$ and $u_{h, k}$. In this section, we analyze the error between $u$ and $u_{h, k}$ measured in the norm of $\mathbb{H}^{r}(\Omega)$, for $r \in[0,1]$.

The following theorem provides conditions on $f$ which imply exponential convergence of the error $\left\|u_{h}-u_{h, k}\right\|_{\mathbb{H}^{r}(\Omega)}$.

Theorem 4.2. Let $r$ be in $[0,1]$ and $N$ and $M$ be as in (3.1). Then for $\beta>r / 2$,

$$
\left\|u_{h}-u_{h, k}\right\|_{\mathbb{H}^{r}(\Omega)} \preceq e^{-\pi^{2} /(2 k)}\|f\|
$$

and for $\beta \leq r / 2$ and $f \in \mathbb{H}^{r-2 \beta+\epsilon}(\Omega)$ satisfying $r-2 \beta+\epsilon \in[0,1+\alpha]$,

$$
\left\|u_{h}-u_{h, k}\right\|_{\mathbb{H}^{r}(\Omega)} \preceq e^{-\pi^{2} /(2 k)}\|f\|_{\mathbb{H}^{r-2 \beta+\epsilon}(\Omega)} .
$$


Proof. Applying (34) gives

$$
\left\|u_{h}-u_{h, k}\right\|_{\mathbb{H}^{r}(\Omega)} \preceq\left\|A_{h}^{r / 2}\left(u_{h}-u_{h, k}\right)\right\| .
$$

If $\beta>r / 2$, we apply Theorem 3.2 with $s=r / 2$ and $t=0$ to obtain

$$
\left\|A_{h}^{r / 2}\left(u_{h}-u_{h, k}\right)\right\| \preceq e^{-\pi^{2} /(2 k)}\left\|\pi_{h} f\right\| \preceq e^{-\pi^{2} /(2 k)}\|f\| .
$$

Alternatively, when $\beta \leq r / 2$, we note that $t:=(r+\epsilon) / 2-\beta$ is in $[0,(1+\alpha) / 2]$ and $s:=\beta-\epsilon / 2<\beta$. Now, applying Theorem 3.2. Lemma 4.1. (34) and (31) gives

$$
\left\|A_{h}^{r / 2}\left(u_{h}-u_{h, k}\right)\right\| \preceq e^{-\pi^{2} /(2 k)}\left\|\pi_{h} f\right\|_{D\left(A_{h}^{t}\right)} \preceq e^{-\pi^{2} /(2 k)}\|f\|_{\mathbb{H}^{2 t}(\Omega)} .
$$

Combining the above three inequalities completes the proof of the theorem.

We next discuss the error bounds for $\left\|u-u_{h}\right\|_{\mathbb{H}^{r}(\Omega)}$ given in Theorem 6.2 of [10] with $r \in[0,1]$. The proof given there uses both (32) and (33) with of $r_{1}=\alpha$ and $r_{2}=\min \{\alpha, 1-r\}$ and results in an order of convergence $0<2 \alpha^{*}=r_{1}+r_{2}$ (with possibly a logarithm of $h^{-1}$ deterioration depending on the regularity of $f$ ).

Theorem 6.2 of $[10$ shows that:

Case 1: When $r / 2+\alpha^{*}-\beta \geq 0$ and $f$ is in $D\left(A^{r / 2+\alpha^{*}-\beta}\right)$

$$
\begin{aligned}
\left\|u-u_{h}\right\|_{\mathbb{H}^{r}(\Omega)} & \preceq \log \left(h^{-1}\right) h^{2 \alpha^{*}}\|f\|_{D\left(A^{r / 2+\alpha^{*}-\beta}\right)} \\
& \preceq \log \left(h^{-1}\right) h^{2 \alpha^{*}}\|f\|_{\mathbb{H}^{r+2 \alpha^{*}-2 \beta}(\Omega)} .
\end{aligned}
$$

Case 2: When $r / 2+\alpha^{*}-\beta \geq 0$ and $f$ is in $D\left(A^{r / 2+\alpha^{*}-\beta+\epsilon}\right)$ with $r+2 \alpha^{*}-2 \beta+2 \epsilon \leq$ $1+\alpha$

$$
\left\|u-u_{h}\right\|_{\mathbb{H}^{r}(\Omega)} \preceq h^{2 \alpha^{*}}\|f\|_{D\left(A^{\left.r / 2+\alpha^{*}-\beta+\epsilon\right)}\right.} \preceq h^{2 \alpha^{*}}\|f\|_{\mathbb{H}^{r+2 \alpha^{*}-2 \beta+2 \epsilon}(\Omega)} .
$$

Case 3: When $r / 2+\alpha^{*}-\beta<0$ and $f \in L^{2}(\Omega)$,

$$
\left\|u-u_{h}\right\|_{\mathbb{H}^{r}(\Omega)} \preceq h^{2 \alpha^{*}}\|f\| .
$$

The following theorem follows from combining the above results with Theorem 4.2

Theorem 4.3. For $r \in[0,1], k>0$ and $N$ and $M$ be as in (3.1). Then we have

$$
\left\|u-u_{h, k}\right\|_{\mathbb{H}^{r}(\Omega)} \preceq \begin{cases}\left(\log \left(h^{-1}\right) h^{2 \alpha_{*}}+e^{-\pi^{2} /(2 k)}\right)\|f\|_{\mathbb{H}^{r+2 \alpha^{*}-2 \beta}(\Omega)} & \text { in Case 1, } \\ \left(h^{2 \alpha_{*}}+e^{-\pi^{2} /(2 k)}\right)\|f\|_{\mathbb{H}^{r+2 \alpha^{*}-2 \beta+2 \epsilon}(\Omega)} & \text { in Case 2, } \\ \left(h^{2 \alpha_{*}}+e^{-\pi^{2} /(2 k)}\right)\|f\| & \text { in Case 3. }\end{cases}
$$

Proof. We set $C_{1}:=\|f\|_{\mathbb{H}^{r+2 \alpha^{*}-2 \beta}(\Omega)}, C_{2}=\|f\|_{\mathbb{H}^{r+2 \alpha^{*}-2 \beta+2 \epsilon}(\Omega)}$ and $C_{3}=\|f\|$. We note that

$$
\|f\|_{\mathbb{H}^{s}(\Omega)} \leq\|f\|_{\mathbb{H}^{t}(\Omega)}, \text { for } 0 \leq s \leq t \leq 2 \text { and } f \in \mathbb{H}^{t}(\Omega) .
$$

This means that $\|f\| \leq C_{j}$, for $j=1,2,3$. Thus, when $\beta>r / 2$, Theorem 4.2 yields

$$
\left\|u_{h}-u_{h, k}\right\|_{\mathbb{H}^{r}(\Omega)} \preceq C_{j} e^{-\pi^{2} /(2 k)}, \quad \text { for } j=1,2,3 .
$$

When $\beta \leq r / 2$, we are in Cases 1 or 2 and Theorem 4.2 with $\epsilon=2 \alpha^{*}$ together with (37) imply

$$
\left\|u_{h}-u_{h, k}\right\|_{\mathbb{H}^{r}(\Omega)} \leq e^{-\pi^{2} /(2 k)}\|f\|_{\mathbb{H}^{r-2 \beta+2 \alpha^{*}}} \leq C_{j} e^{-\pi^{2} /(2 k)},
$$

for $j=1$ or $j=2$. The desired result follows from the above estimates, the bounds for $\left\|u-u_{h}\right\|_{\mathbb{H}^{r}(\Omega)}$ above and the triangle inequality. 


\section{Numerical ILlustration.}

In this section, we present some numerical experiments to illustrate the error estimates derived in Section 3. In order for the error due to the finite element approximation to shadow the exponentially converging sinc quadrature error, we consider the following one dimensional problem:

$$
\begin{aligned}
& A^{\beta} u=1, \quad \text { in }(0,1), \\
& u(0)=u(1)=0,
\end{aligned}
$$

where $A$ is an unbounded operator associated with the bilinear form

$$
A(u, v)=\int_{0}^{1} u^{\prime} v^{\prime} d x \quad \text { for } u, v \in H_{0}^{1}(0,1)
$$

with $H_{0}^{1}(0,1):=\left\{v \in H^{1}(0,1): v(0)=v(1)=0\right\}$. In particular, we provide numerical evidence of exponential rates not explained by the previous theory in 10. but by Theorem 3.2. Finally, we refer to 9,10 for numerical experiments with domains $\Omega \subset \mathbb{R}^{d}, d>1$, and general operators $A$.

5.1. Error from the Sinc Approximation. We first report the sinc approximation error $\left\|\left(A_{h}^{-\beta}-Q_{k}^{-\beta}\left(A_{h}\right)\right) \pi_{h} f\right\|_{\mathbb{H}^{r}(\Omega)}$ for $r \in[0,1]$. To this end, we consider a subdivision $\mathcal{T}_{h}$ made of uniform intervals of length $h=1 / 512$. The finite element approximation of $u$ in 38 is given by

$$
u_{h}=A_{h}^{-\beta} \pi_{h} f=\sum_{\ell=1}^{D_{h}} \lambda_{\ell, h}^{-\beta}\left(1, \psi_{\ell, h}\right) \psi_{\ell, h},
$$

where the number of degrees of freedom $D_{h}=511$ and $\left\{\psi_{\ell, h}, \lambda_{\ell, h}\right\}$ are the eigenpairs of $A_{h}$, i.e.

$$
\lambda_{\ell, h}=\frac{6(1-\cos (k \pi h))}{h^{2}(2+\cos (k \pi h))} \text { and } \psi_{\ell, h}=\sqrt{\frac{6}{2+\cos (h l \pi)}} \sum_{k=1}^{D_{h}} \sin (h \ell k \pi) \varphi_{k, h} .
$$

Similarly, the sinc approximation of $u_{h}$ is given by

$$
u_{h, k}=Q_{k}^{-\beta}\left(A_{h}\right) \pi_{h} f=\sum_{\ell=1}^{D_{h}} \mathcal{Q}_{k}^{-\beta}\left(\lambda_{\ell, h}\right)\left(1, \psi_{\ell, h}\right) \psi_{\ell, h} .
$$

We report the errors in the discrete operator norm (see, (34)), namely

$$
e(k, r):=\left\|u_{h}-u_{h, k}\right\|_{D\left(A_{h}^{r / 2}\right)}=\left(\sum_{l=1}^{D_{h}} \lambda_{\ell, h}^{r}\left|\left(u_{h}-u_{h, k}, \psi_{\ell, h}\right)\right|^{2}\right)^{1 / 2} .
$$

Theorem 3.2 guarantees that $e(k, r) \leq C e^{-c / k}$ for some constants $c$ and $C$ independent of $k$ and $h$. To illustrate this behavior, we provide semi-log plots of the error as a function of $1 / k$ so that $e^{-c / k}$ ends up being a straight line with slope $-c$. Figure 1 reports the values of $e(k, r)$ for $\beta=0.3,0.5,0.7$ and $r=0, \beta, 1$. In this case, $M$ and $N$ are chosen to balance the three error terms coming from the sinc quadrature analysis (see, Remark 3.1). The results are close to straight lines which is consistent with the $C e^{-c / k}$ error behavior of the theory. Since $1 \in H^{1 / 2-\epsilon}(\Omega)$ for any $\epsilon>0$, the exponential rate observed for $r=1$ is not explained by the previous theory in [10] but results from Theorem 3.2 above. 
To illustrate the benefit in choosing the balanced scheme, we provide similar results in Figure 2 for $M=N=1 / k^{2}$. Although, both strategies yield exponential decay, the balanced scheme is dramatically more efficient.
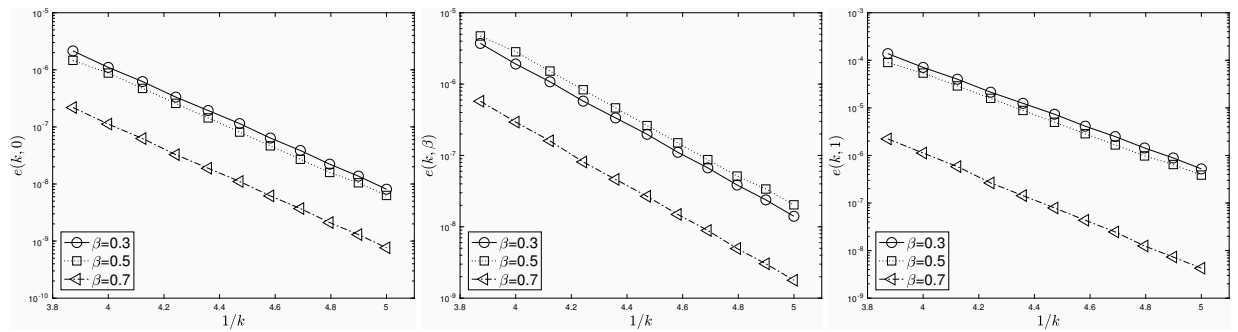

Figure 1. Values of $e(k, r)$ defined by (41) for different sinc quadrature spacing $k$ and $r=0$ (left), $r=\beta$ (middle) and $r=1$ (right). Here $M$ and $N$ are chosen to balance the three error terms coming from the sinc quadrature $\mathbb{H}^{r}(\Omega)$ for a given sinc quadrature spacing $k$ (see, Remark 3.1).
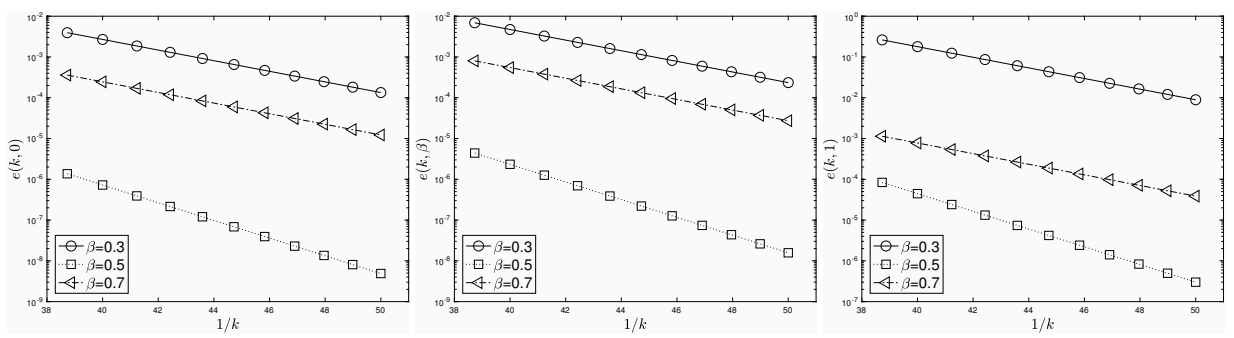

Figure 2. Values of $e(k, r)$ defined by (41) for different sinc quadrature spacing $k$ and $r=0$ (left), $r=\beta$ (middle) and $r=1$ (right). In contrast with the experiments provided in Figure 1 . here $M=N=1 / k^{2}$.

5.2. Total Error. We compare the solution $u$ in $(38)$ and its fully discrete approximation $u_{h, k}$ given by (40). The sequence of meshes are obtained upon performing successive uniform refinements of the unit interval, thereby leading to mesh sizes $h_{j}=2^{-j}$ for $j=3, \ldots, 8$.

Since $1 \in H^{1 / 2-\epsilon}(\Omega)$ for any $\epsilon>0$, the predicted rate of convergence (up to a logarithmic term) is described by

$$
\left\|u-u_{h, k}\right\| \preceq h^{\max \{2,2 \beta+1 / 2\}}
$$

upon setting

and by

$$
k=\frac{1}{(8(2 \beta+1 / 2) \log (1 / h)}
$$

$$
\left\|u-u_{h, k}\right\|_{H^{1}(\Omega)} \preceq h^{\max \{2,2 \beta+1 / 2\}-1}
$$


with the choice

$$
k=\frac{1}{(4(2 \beta-1 / 2) \log (1 / h))} .
$$

We note that the constant 8 and 4 appearing in the choices of $k$ are tuned so that the error of the sinc quadrature is already in its asymptotic regime for small $k$. As already noted (see Figure 1 and 2), this choice is not necessary for the algorithm to work.

Also, the solution $u$ is not known exactly and is therefore approximated by truncating its eigenfunction expansion

$$
u \approx 2 \sum_{\ell=1}^{50000}(\pi \ell)^{-\beta} \frac{1-(-1)^{\ell}}{\pi \ell} \sin (\pi \ell x) .
$$

The errors between $u$ and $u_{h, k}$ are reported in Figure 3 and matches the predictions of Theroem 4.3 .
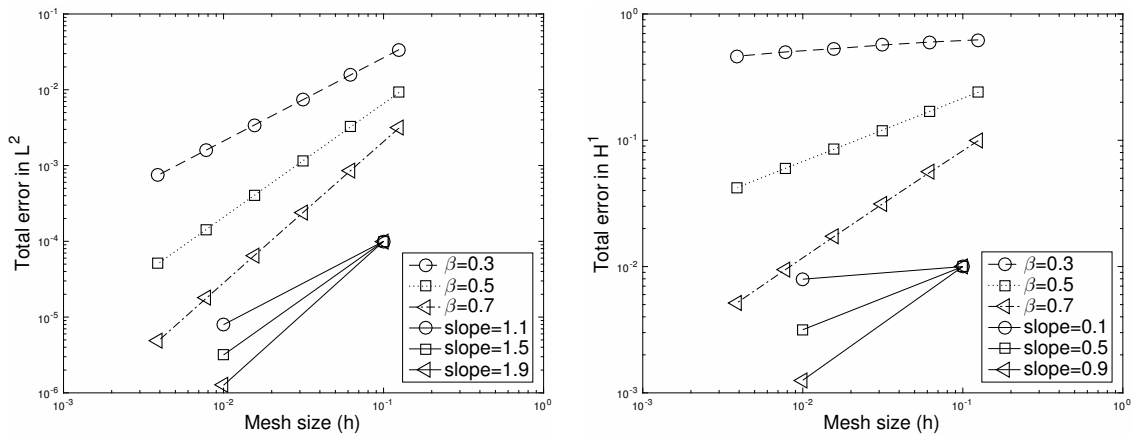

FiguRE 3. Errors between $u$ and $u_{h, k}$ in the $L^{2}(\Omega)$ norm (left) and the $H^{1}(\Omega)$ norm (right) versus the mesh size for $\beta=0.3,0.5,0.7$. The rates of convergences predicted by $\sqrt[42]{4}$ and $\sqrt{43}$ are observed numerically.

\section{REFERENCES}

[1] Gabriel Acosta and Juan Pablo Borthagaray, A fractional Laplace equation: regularity of solutions and finite element approximations, SIAM J. Numer. Anal. 55 (2017), 472-495.

[2] M. S. Agranovich and A. M. Selitskiü, Fractional powers of operators corresponding to coercive problems in Lipschitz domains, Funktsional. Anal. i Prilozhen. 47 (2013), 2-17.

[3] Harbir Antil, Johannes Pfefferer and Sergejs Rogovs, Fractional Operators with Inhomogeneous Boundary Conditions: Analysis, Control, and Discretization, arXiv preprint arXiv:1703.05256 (2017).

[4] A. V. Balakrishnan, Fractional powers of closed operators and the semigroups generated by them, Pacific J. Math. 10 (1960), 419-437.

[5] Lehel Banjai, Jens M Melenk, Ricardo H Nochetto, Enrique Otarola, Abner J Salgado and Christoph Schwab, Tensor FEM for spectral fractional diffusion, arXiv preprint arXiv:1707.07367 (2017).

[6] Randolph E. Bank and Harry Yserentant, On the $H^{1}$-stability of the $L_{2}$-projection onto finite element spaces, Numer. Math. 126 (2014), 361-381.

[7] Andrea Bonito, Juan Pablo Borthagaray, Ricardo H Nochetto, Enrique Otarola and Abner J Salgado, Numerical methods for fractional diffusion, arXiv preprint arXiv:1707.01566 (2017).

[8] Andrea Bonito, Wenyu Lei and Joseph E Pasciak, Numerical approximation of the integral fractional Laplacian, arXiv preprint arXiv:1707.04290 (2017). 
[9] Andrea Bonito and Joseph E. Pasciak, Numerical approximation of fractional powers of elliptic operators, Math. Comp. 84 (2015), 2083-2110.

[10] Andrea Bonito and Joseph E. Pasciak, Numerical approximation of fractional powers of regularly accretive operators, IMA J. Numer. Anal. 37 (2017), 1245-1273.

[11] James H. Bramble, Joseph E. Pasciak and Olaf Steinbach, On the stability of the $L^{2}$ projection in $H^{1}(\Omega)$, Math. Comp. 71 (2002), 147-156.

[12] M. Crouzeix and V. Thomée, The stability in $L_{p}$ and $W_{p}^{1}$ of the $L_{2}$-projection onto finite element function spaces, Math. Comp. 48 (1987), 521-532.

[13] Marta D'Elia and Max Gunzburger, The fractional Laplacian operator on bounded domains as a special case of the nonlocal diffusion operator, Comput. Math. Appl. 66 (2013), 12451260.

[14] Alexandre Ern and Jean-Luc Guermond, Theory and practice of finite elements, Applied Mathematical Sciences 159, Springer-Verlag, New York, 2004.

[15] M. Ilic, F. Liu, I. Turner and V. Anh, Numerical approximation of a fractional-in-space diffusion equation. I, Fract. Calc. Appl. Anal. 8 (2005), 323-341.

[16] M. Ilic, F. Liu, I. Turner and V. Anh, Numerical approximation of a fractional-in-space diffusion equation. II. With nonhomogeneous boundary conditions, Fract. Calc. Appl. Anal. 9 (2006), 333-349.

[17] Tosio Kato, Fractional powers of dissipative operators, J. Math. Soc. Japan 13 (1961), 246274.

[18] Alessandra Lunardi, Interpolation theory, second ed, Appunti. Scuola Normale Superiore di Pisa (Nuova Serie). [Lecture Notes. Scuola Normale Superiore di Pisa (New Series)], Edizioni della Normale, Pisa, 2009.

[19] John Lund and Kenneth L. Bowers, Sinc methods for quadrature and differential equations, Society for Industrial and Applied Mathematics (SIAM), Philadelphia, PA, 1992.

[20] Dominik Meidner, Johannes Pfefferer, Klemens Schürholz and Boris Vexler, $h p$-Finite Elements for Fractional Diffusion, arXiv preprint arXiv:1706.04066 (2017).

[21] Ricardo H. Nochetto, Enrique Otárola and Abner J. Salgado, A PDE approach to fractional diffusion in general domains: a priori error analysis, Found. Comput. Math. 15 (2015), 733791.

[22] Qianqian Yang, Ian Turner, Fawang Liu and Milos Ilić, Novel numerical methods for solving the time-space fractional diffusion equation in two dimensions, SIAM J. Sci. Comput. 33 (2011), 1159-1180.

(A. Bonito) Department of Mathematics, Texas A\&M University, College Station, TX 77843, USA

E-mail address: bonito@math.tamu.edu

(W. Lei) Department of Mathematics, Texas A\&M University, College Station, TX 77843 , USA

E-mail address: wenyu@math.tamu.edu

(J. Pasciak) Department of Mathematics, Texas A\&M University, College Station, TX 77843 , USA

E-mail address: pasciak@math.tamu.edu 